\title{
Soliton pattern formation in figure-of-eight laser
}

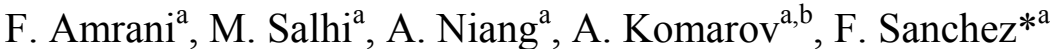 \\ ${ }^{a}$ Laboratoire de Photonique E.A. 4464, Université d'Angers, 2 Bd Lavoisier, 49000 Angers, France \\ ${ }^{\mathrm{b}}$ Institute of Automation and Electrometry, Russian Academy of Sciences, Acad. Koptyug Pr. 1, \\ 630090 Novosibirsk, Russia
}

\begin{abstract}
We investigate the soliton pattern formation in an erbium-doped figure-of-eight double-clad fiber laser. The modelocking is realized with a nonlinear amplifying loop mirror. Different soliton complexes have been obtained similar to those obtained when the mode-locking is achieved through the nonlinear polarization rotation technique.
\end{abstract}

Keywords: fiber laser, mode-locking, soliton, short-pulse, interaction

\section{INTRODUCTION}

Passively mode-locked fiber lasers have demonstrated their ability to generate several types of soliton complexes, especially in the anomalous dispersion regime where the soliton energy quantization favours multiple pulsing [1]. In particular, bound states of few pulses have been observed independently of both the dispersion regime and the exact physical mechanism of mode-locking. For example, bound states have been reported in ring cavities passively modelocked through nonlinear polarization rotation (NLPR) [2,3] and in figure-of-eight lasers (F8L) where mode locking is achieved with either a nonlinear amplifying loop mirror (NALM) [4] or a nonlinear optical loop mirror (NOLM) [5]. As mentioned by several authors [6], bound states are an intrinsic feature of fiber lasers and can be considered as a universal dynamical behaviour. With this in mind, it is not surprising that historically, bound states have been first predicted with models based on universal equations which do not consider the exact physical mechanism of mode-locking, such as various versions of the Ginzburg-Landau equation $[7,8]$. The scaling up of the output power of fiber lasers in recent years has allowed to considerably increase the number of coexisting solitons in passively mode-locked fiber lasers. Thus the number of interacting pulses has undergone a big step from a few tens to several hundreds, resulting in new and fascinating complex pattern formation dynamics. Concerning such large numbers of self-organized dissipative solitons, recent results have been obtained using the nonlinear polarization rotation technique to passively mode lock the fiber laser. A bound state containing several hundreds of pulses was first reported in [9]. By analogy with an atomic lattice, and following the initial suggestion of Mitschke [10], this state was named a soliton crystal. More recently, several ordered and disordered patterns presenting analogy with the states of matter were observed [11]. A reconstruction has been performed in [12] for a soliton gas, a soliton liquid, a soliton polycrystal and a soliton crystal. From the theoretical point of view, there are only few predictions concerning the self-organization of a very large assembly of dissipative optical solitons. A model based on a modified cubic Ginzburg-Landau equation had been proposed to describe the soliton crystal formation of infinite extent [13]. An alternative model [1], which reduces to the quintic Ginzburg-Landau equation in the limit of small intensities [14], allowed the description of a soliton crystal of finite extent [15]. The fact that averaged models support soliton crystal solutions suggests that such organized state could be an intrinsic feature of high power fiber lasers independently of the exact mode-locking mechanism. This was the initial motivation of the work reported in this paper. We have realized a figure-of-eight fiber laser showing that this configuration can generate a soliton gas, a soliton liquid, a soliton polycrystal and a soliton crystal, thus demonstrating the universality of such soliton patterns [16]. In addition, an intricate state involving a mixture of crystal and liquid soliton phases is also observed [17].

\section{EXPERIMENTAL RESULTS}

\subsection{Experimental setup}

The experimental setup is schematically represented in Fig. 1. It is an all-fiber figure-of-eight laser based on a passive

*francois.sanchez@univ-angers.fr; phone 3324173 54 47; fax 33242735216

Nonlinear Optics and Applications VI, edited by Benjamin J. Eggleton, Alexander L Gaeta, Neil G. Broderick, Proc. of SPIE Vol. 8434, 843403 - @ 2012 SPIE · CCC code: 0277-786X/12/\$18 · doi: 10.1117/12.919994 
unidirectional ring (UR) cavity that is coupled to a NALM through a 50/50 fiber coupler. The NALM contains a doubleclad Er:Yb-doped fiber amplifier manufactured by Keopsys, which consists of a 2.45-meter long double-clad fiber (DCF) that has a chromatic dispersion coefficient $\beta_{2}^{\mathrm{DCF}}=-0.0247 \mathrm{ps}^{2} / \mathrm{m}$ at the operating wavelength $\lambda=1.55 \mu \mathrm{m}$, and is pumped at $980 \mathrm{~nm}$ by a $4-\mathrm{W}$ semiconductor laser. Two pieces of single-mode fiber ( $\mathrm{SMF} 28, \beta_{2}^{\mathrm{SMF}}=-0.022 \mathrm{ps}^{2} / \mathrm{m}$ ) and dispersion shifted fiber (DSF, $\beta_{2}^{\mathrm{DSF}}=0.14 \mathrm{ps}^{2} / \mathrm{m}$ ) are used to control both the total cavity dispersion and the nonlinearity experienced by mode-locked pulses as they propagate. To favour multiple-pulse mode locking, the total dispersion is set in the anomalous regime with $\beta_{2}^{\mathrm{TOT}} \mathrm{L} \approx-0.04 \mathrm{ps}^{2}$, with a total cavity length of $27.5 \mathrm{~m}$ corresponding to a round-trip time of $137.6 \mathrm{~ns}$. The UR part is composed of an optical isolator and a $20 \%$-output coupler. For high enough pump power and suitable length of the NALM, mode locking is obtained through the adjustment of the two polarization controllers (PC). The output intensity is detected with a high-speed photodetector (TIA-1200) and visualized with a fast oscilloscope (Tektronix TDS $6124 \mathrm{C}, 12 \mathrm{GHz}, 40 \mathrm{GS} / \mathrm{s}$ ). The spectral properties are analyzed with an optical spectrum analyzer (Anritsu MS 9710C) and the pulse duration is measured with an optical autocorrelator with a scanning range of \pm 100 ps (Femtochrome FR-103 XL).

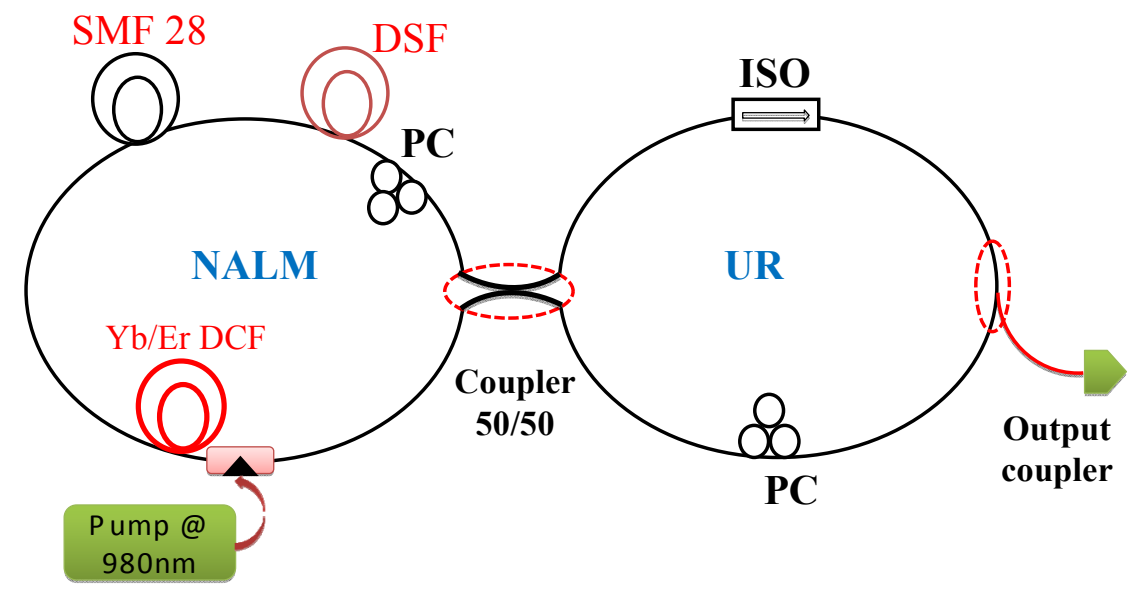

Figure 1. Experimental setup. DCF: double-clad fiber; DSF: dispersion-shifted fiber; ISO: optical isolator; PC: polarization controller. NALM: nonlinear amplifying loop mirror, UR: unidirectional ring.

\subsection{Soliton gas}

Different soliton patterns can be observed by varying the polarization controllers. The most commonly obtained distribution is a state in which hundreds of solitons fill all the available space along the cavity and are in ceaseless relative motions. An example of temporal distribution is given in Fig. 2. The autocorrelation trace shown in Fig. 3(a) exhibits only a central peak with a very large pedestal thus indicating that the solitons are in perpetual relative motion while the optical spectrum of Fig. 3(b) is not modulated suggesting that there is no constant phase relation between the pulses. Fig. 3(b) also exhibits a significant continuous component visible in the centre of the spectrum. This state can be compared to a "soliton gas", as we reported in NLPR-based fiber lasers [11].

\subsection{Soliton liquid}

Adjusting the polarization controllers allows switching to a condensed phase that fills about $8 \%$ of the cavity, as shown in Fig. 4(a). Although incompletely resolved, real-time recording reveals internal motions of solitons inside the condensed phase that can here be compared to a liquid [11,12]. Note that the increasing average level of the temporal trace is an artifact due to our photodetector. Additional insight is obtained from the autocorrelation trace shown in Fig. 4(b) and from the optical spectrum (not shown). The regular spacing of the peaks in the autocorrelation trace indicates an average pulse-to-pulse separation of $11 \mathrm{ps}$ inside packets comprising around 12 solitons. Whereas the total number of 
solitons is estimated to be as large as 800 , these soliton packets are similar to micro-droplets having relative freedom of motion inside the condensed phase. A detailed inspection of Fig. 4(b) indicates that the width of the central peak (4 ps) is significantly smaller than the width of the peaks located at the edge of the scanning range $(5.5 \mathrm{ps})$. This indicates a slightly non-uniform temporal distribution of pulses inside droplets. The pedestal of the autocorrelation trace is also pointing out the lack of a precise separation between all pulses. Finally, the absence of modulation in the spectrum suggests that there is no stable phase relationship between pulses from one round trip to the next.

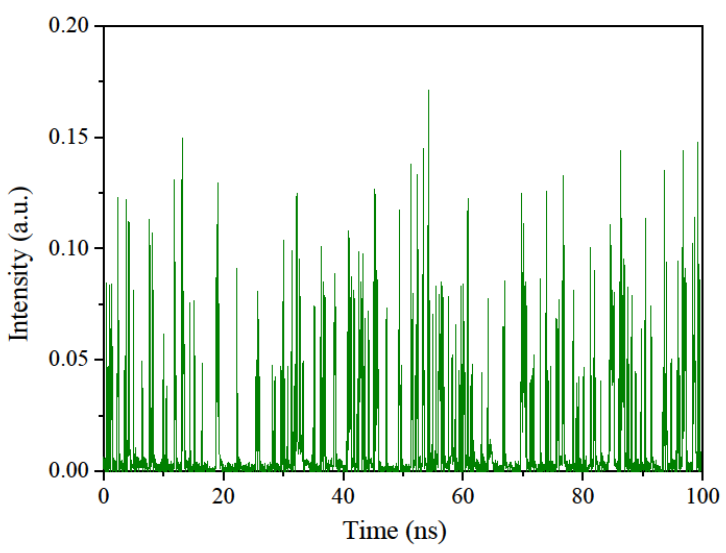

Figure 2. Temporal distribution of a soliton gas.
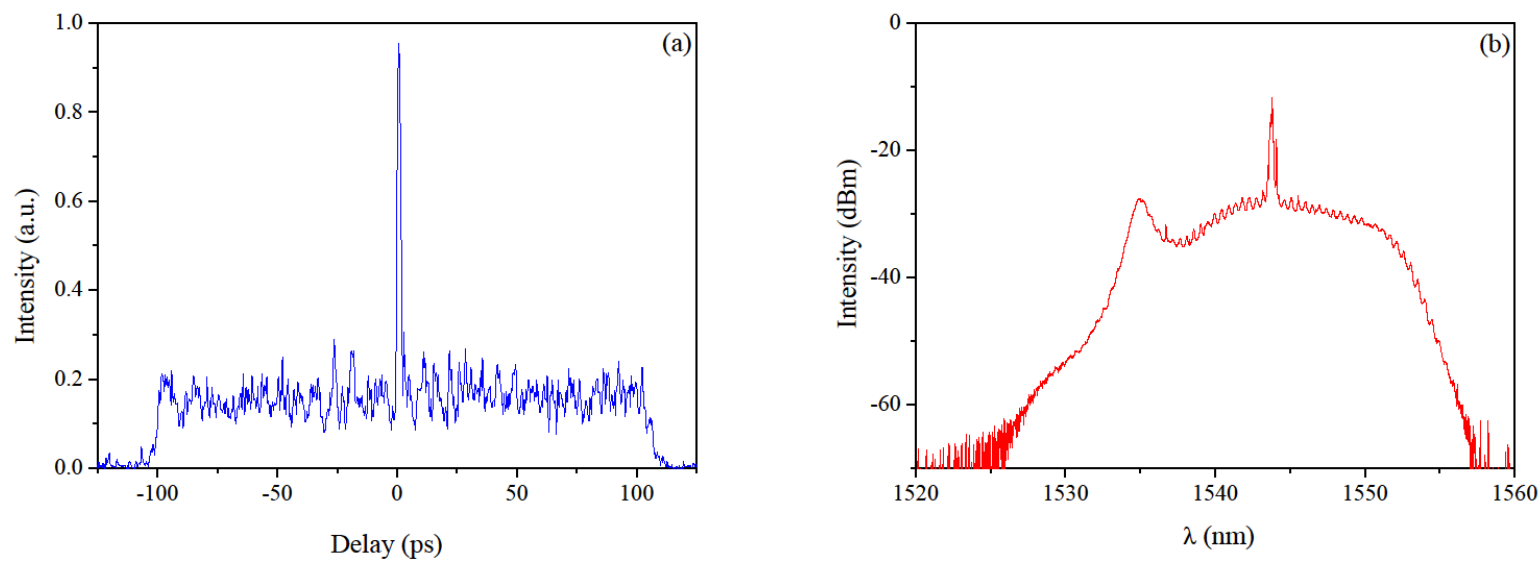

Figure 3. (a) Autocorrelation trace and (b) optical spectrum of a soliton gas.
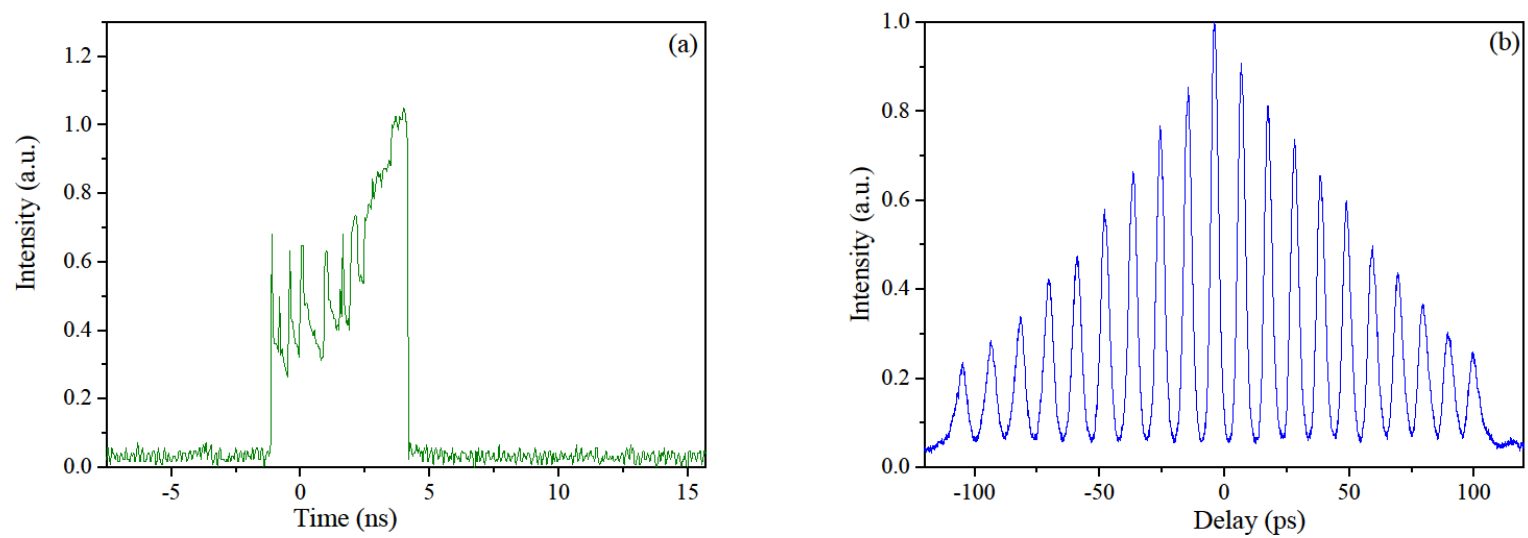

Figure 4. (a) Temporal trace and (b) autocorrelation trace of a soliton liquid. 


\subsection{Soliton polycrystal}

By slightly varying the polarization controllers we obtained the temporal distribution given in Fig. 5. It consists in a very stable distribution of seven well-separated soliton packets that contain nearly the same amount of solitons. The optical spectrum (Fig. 6(b)) exhibits a strong modulation revealing the high degree of correlation between pulses inside each packet. The spectral period is $2 \mathrm{~nm}$ leading to a temporal delay between neighbouring pulses of $4.7 \mathrm{ps}$, as confirmed by the pedestal-free autocorrelation trace of Fig. 6(a). The nearly triangular envelope of the autocorrelation trace, all peaks beneath having the same 1.5-ps width, is characteristic of bound-states of regularly-spaced solitons, and its prolongation till the autocorrelation background indicates a number of roughly 30 bound solitons. The overall soliton distribution inside the cavity is therefore an incoherent sequence of small soliton crystals, or crystallites. This soliton pattern is close to the one reported in $[11,12]$ except that the crystallites are almost identical and well separated inside the cavity.

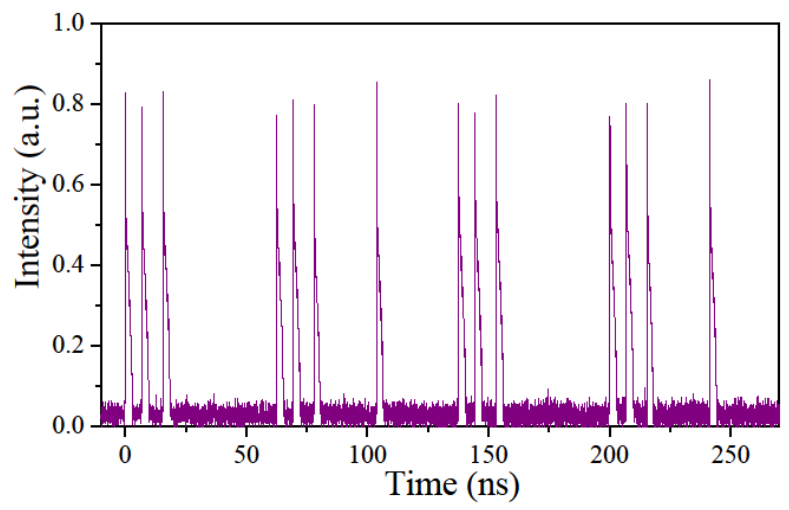

Figure 5. Temporal trace of a soliton polycristal.
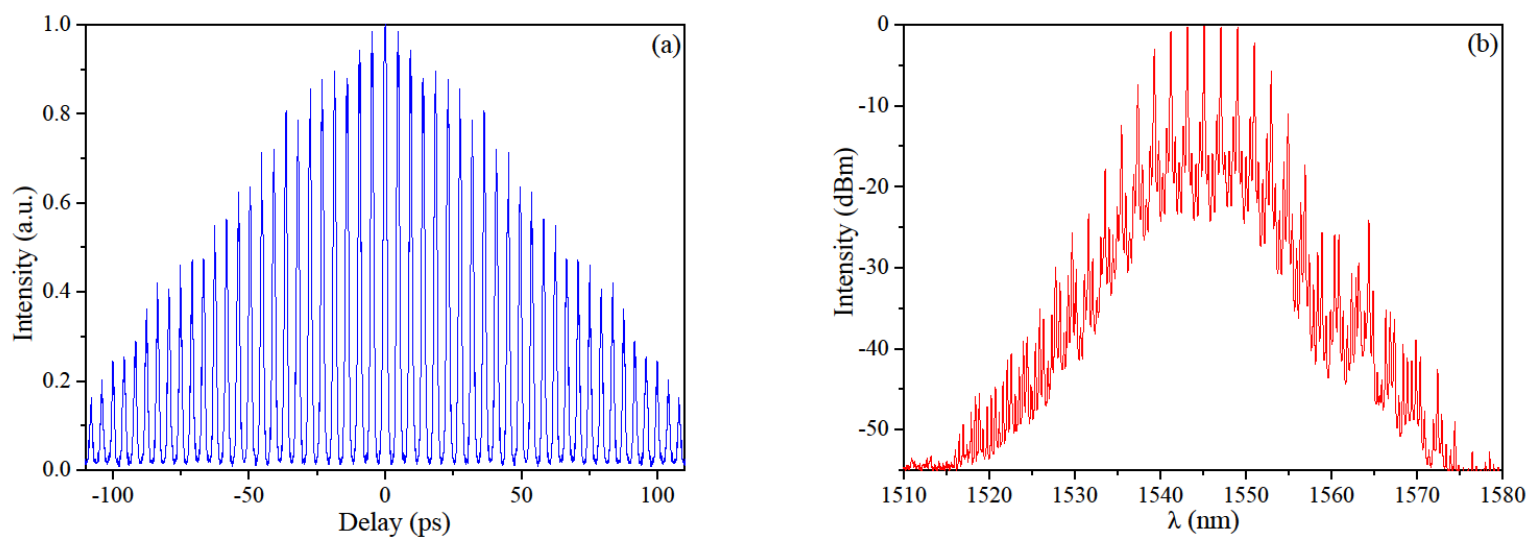

Figure 6. (a) Autocorrelation trace and (b) optical spectrum of a soliton polycrystal..

\subsection{Soliton crystal}

This state is remarkable as it consists of a large number of regularly-spaced solitons with constant relative phase differences from one round trip to the next. It is a bound-state of several hundreds of solitons, or soliton crystal, similar to the cases recently reported in NLPR-based passively mode-locked double-clad fiber lasers $[9,11,13]$. The soliton train spans over $7 \mathrm{~ns}$, as indicated by the temporal intensity recording of Fig. 7. Note that the increasing average level of the temporal trace is an artifact due to our photodetector. However, the corresponding optical spectrum given in Fig. 8(b) proves the strong coherence between pulses since it is modulated with a contrast exceeding $95 \%$. The spectral period is $0.552 \mathrm{~nm}$ which corresponds to a temporal separation of $14.5 \mathrm{ps}$ between solitons, in good agreement with the 
autocorrelation trace shown in Fig. 8(a), which is mainly composed of 1.5-ps-wide equidistant peaks. From the train duration and the delay between solitons, we estimate the number of pulses in the soliton crystal to be 482 .

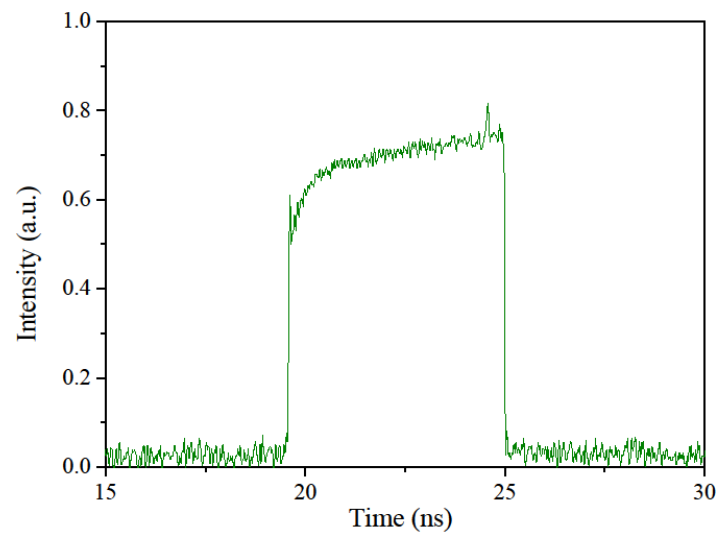

Figure 7. Temporal trace of a soliton crystal.
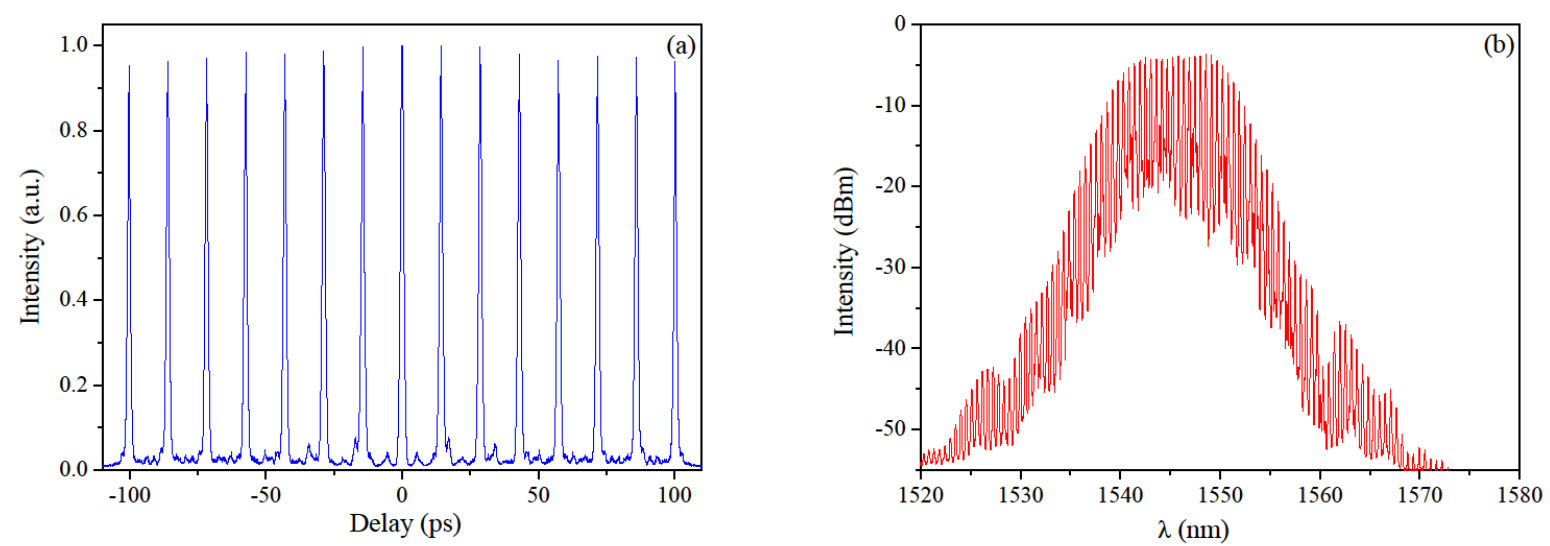

Figure 8. (a) Autocorrelation trace and (b) optical spectrum of a soliton crystal.

\subsection{Intricate state}

For a pump power of $3.2 \mathrm{~W}$ and an adequate adjustment of polarization controllers, we obtain the temporal distribution given in Fig. 9 [17]. The state consists of a set of confined pulses occupying a small part of the cavity. A more detailed inspection (zoom of Figure 2) shows that there is an almost periodic series of peaks followed by plateaus. A peak with a width of $50 \mathrm{ps}$ and a plateau with a width of $340 \mathrm{ps}$ repeat several times to form a block of about $7 \mathrm{~ns}$. The experiment shows that the solitons in the peaks are at rest while those of the plateaus are subject to fluctuations in amplitude suggesting that solitons move. Note that the increasing level of the plateaus is an artifact due to our photodetector. The peaks are therefore in a solid state (glass or crystal) while the plateaus are in liquid state. The autocorrelation trace of Fig. 10(a) suggests that, inside the peaks, the solitons could have some phase correlation and form a microcrystal. However no clear modulation is visible in the optical spectrum of Fig.10(b). Indeed, the spectrum appears more noisy than modulated. The resolution of our oscilloscope was $50 \mathrm{ps}$ and does not allow determining the number of pulses within each state. However, from the autocorrelation trace, we can deduce the distance between pulses in the crystal phase, which is about $7 \mathrm{ps}$.

\section{CONCLUSION}

In summary, we have obtained in the figure-of-eight double-clad fiber laser a variety of soliton patterns involing a large number of pulses. Some of these patterns were previously observed in double-clad fiber lasers passively mode-locked 
through nonlinear polarization rotation. More precisely, we have obtained many-soliton states which, by analogy with the states of matter, can be dubbed as a soliton gas, soliton liquid, soliton crystal and polycrystal. Our results tend to demonstrate, for the first time, the universality of such soliton states. Indeed, the patterns do not depend on the precise mode-locking mechanism. This is a significant step which provides incentive to developing theoretical approaches based on general dynamical models rather than detailed propagation models meant for a specific subset of mode-locked laser cavities. In addition, we have reported an intricate soliton state containing a quasi-periodic series of alternate crystal and liquid soliton phases.

We thank the Agence Nationale de la Recherche for supporting this work (Contract ANR-2010-BLANC-0417-01SOLICRISTAL).

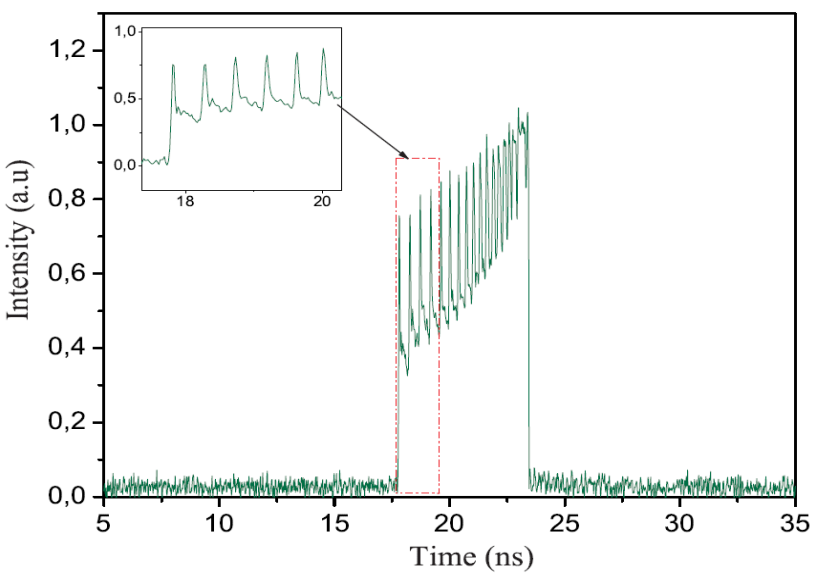

Figure. 9. Temporal trace of the soliton distribution.
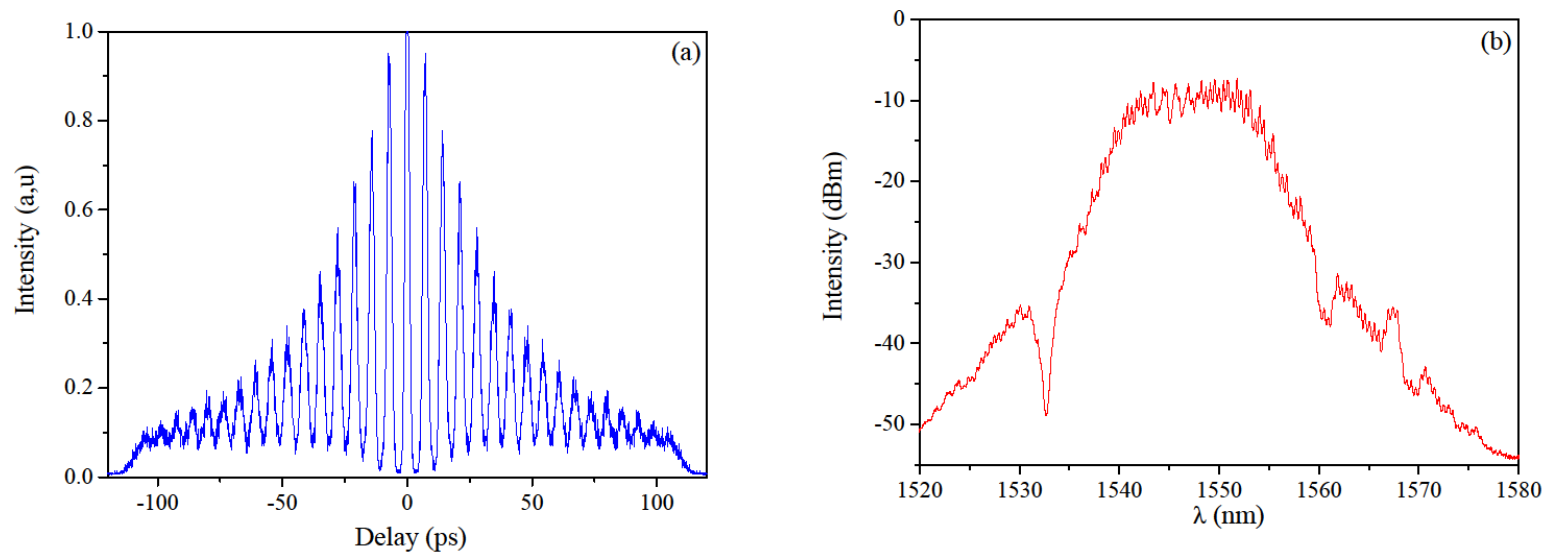

Figure 10. Diphasic state. (a) Autocorrelation trace. (b) Optical spectrum.

\section{REFERENCES}

[1] A. Komarov, H. Leblond and F. Sanchez, "Multistability and hysteresis phenomena in passively mode-locked fiber lasers", Phys. Rev. A 71, 053809 (2005).

[2] D. Y. Tang, W. S. Man, H. Y. Tam and P. D. Drummond, "Observation of bound states of solitons in a passively mode-locked fiber laser”, Phys. Rev. A 64, 033814 (2001). 
[3] Ph. Grelu, F. Belhache, F. Gutty and J. M. Soto-Crespo, "Phase-locked soliton pairs in a stretched-pulsed fiber laser", Opt. Lett. 27, 966 (2002).

[4] M. J. Guy, D. U. Noske, and J. R. Taylor, "Generation of femtosecond soliton pulses by passive mode locking of an ytterbium-erbium figure-of-eight fiber laser", Opt. Lett. 18, 1447 (1993).

[5] N. H. Seong and Dug Y. Kim, "Experimental observation of stable bound solitons in a figure-eight fiber laser". Opt. Lett. 27. 1321 (2002).

[6] J.M. Soto-Crespo and Ph. Grelu, "Temporal multi-soliton complexes generated by passively mode-locked lasers", Lect. Notes Phys. 661, 207-239 (2005).

[7] B. A. Malomed, "Bound solitons in the nonlinear Schrödinger-Ginzburg-Landau equation", Phys. Rev. A. 44, 6954 (1991)

[8] N. N. Akhmediev, A. Ankiewicz and J. M. Soto-Crespo, "Multisoliton solutions of the complex GinzburgLandau equation", Phys. Rev. Lett. 79, 4047 (1997).

[9] A. Haboucha, H. Leblond, M. Salhi, A. Komarov and F. Sanchez, "Coherent pattern soliton formation in a fiber laser", Opt. Lett. 33, 524 (2008).

[10] B. A. Malomed, A. Schwache and F. Mitschke, "Soliton lattice and gas in passive fiber-ring resonators", Fib. Int. Opt. 17, 267 (1997).

[11]F. Amrani, A. Haboucha, M. Salhi, H. Leblond, A. Komarov and F. Sanchez, "Dissipative solitons compounds in a fiber laser: Analogy with the states of the matter", Appl. Phys. B. 99, 107 (2010).

[12]F. Amrani, M. Salhi, H. Leblond, and F. Sanchez. "Characterization of soliton compounds in a passively modelocked high power fiber laser", Opt. Com, 283, 5224 (2010).

[13] A. Haboucha, H. Leblond, M. Salhi, A. Komarov and F. Sanchez, "Analysis of soliton pattern formation in passively mode-locked fiber lasers", Phys. Rev A. 78, 043806 (2008).

[14] A. Komarov, H. Leblond and F. Sanchez, "Quintic complex Ginzburg-Landau model for ring fiber lasers", Phys. Rev. E 72, 025604(R) (2005).

[15] A. Komarov, A. Haboucha and F. Sanchez, "Ultrahigh repetition rate bound-soliton harmonic passive modelocked laser", Opt. Lett. 33, 2254 (2008).

[16]F. Amrani, M. Salhi, H. Leblond, Ph. Grelu and F. Sanchez, "Universal soliton pattern formation in passively mode-locked fiber lasers", Opt. Lett. 36, 1545 (2011).

[17]F. Amrani, M. Salhi, H. Leblond, A. Haboucha and F. Sanchez, "Intricate solitons state in passively modelocked fiber lasers”, Opt. Express 19, 13134 (2011). 